\title{
Generation of Micro-scale Finite Element Models from Synchrotron X-ray CT Images for Multidirectional Carbon Fibre Reinforced Composites
}

DOI:

10.1016/j.compositesa.2016.09.010

\section{Document Version}

Accepted author manuscript

Link to publication record in Manchester Research Explorer

Citation for published version (APA):

Sencu, R., Wang, Z., Wang, Y., Withers, P., Rau, C., Parson, A., \& Soutis, C. (2016). Generation of Micro-scale Finite Element Models from Synchrotron X-ray CT Images for Multidirectional Carbon Fibre Reinforced Composites. Composites: Part A, 91(1), 85-95. https://doi.org/10.1016/j.compositesa.2016.09.010

\section{Published in:}

Composites: Part A

\section{Citing this paper}

Please note that where the full-text provided on Manchester Research Explorer is the Author Accepted Manuscript or Proof version this may differ from the final Published version. If citing, it is advised that you check and use the publisher's definitive version.

\section{General rights}

Copyright and moral rights for the publications made accessible in the Research Explorer are retained by the authors and/or other copyright owners and it is a condition of accessing publications that users recognise and abide by the legal requirements associated with these rights.

\section{Takedown policy}

If you believe that this document breaches copyright please refer to the University of Manchester's Takedown Procedures [http://man.ac.uk/04Y6Bo] or contact uml.scholarlycommunications@manchester.ac.uk providing relevant details, so we can investigate your claim.

\section{OPEN ACCESS}




\section{Accepted Manuscript}

Generation of Micro-scale Finite Element Models from Synchrotron X-ray CT

Images for Multidirectional Carbon Fibre Reinforced Composites

R.M. Sencu, Z. Yang, Y. Wang, P. Withers, C. Rau, A. Parson, C. Soutis

PII:

S1359-835X(16)30304-9

DOI: http://dx.doi.org/10.1016/j.compositesa.2016.09.010

Reference: JCOMA 4424

To appear in:

Composites: Part A

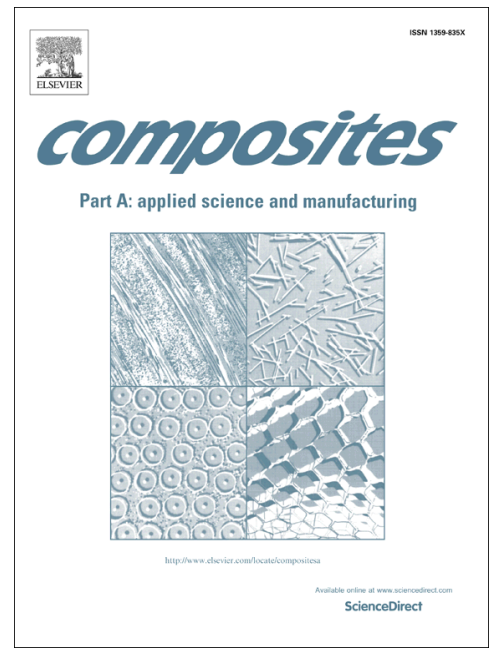

Received Date:

14 April 2016

Revised Date:

5 September 2016

Accepted Date:

10 September 2016

Please cite this article as: Sencu, R.M., Yang, Z., Wang, Y., Withers, P., Rau, C., Parson, A., Soutis, C., Generation of Micro-scale Finite Element Models from Synchrotron X-ray CT Images for Multidirectional Carbon Fibre Reinforced Composites, Composites: Part A (2016), doi: http://dx.doi.org/10.1016/j.compositesa.2016.09.010

This is a PDF file of an unedited manuscript that has been accepted for publication. As a service to our customers we are providing this early version of the manuscript. The manuscript will undergo copyediting, typesetting, and review of the resulting proof before it is published in its final form. Please note that during the production process errors may be discovered which could affect the content, and all legal disclaimers that apply to the journal pertain. 


\title{
Generation of Micro-scale Finite Element Models from Synchrotron X-ray CT Images for Multidirectional Carbon Fibre Reinforced Composites
}

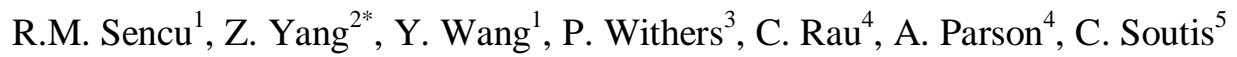 \\ ${ }^{[1]}$ School of Mechanical, Aerospace and Civil Engineering, University of Manchester, M13 9PL, UK \\ ${ }^{[2]}$ Centre for Low Impact Buildings, Faculty of Engineering and Computing, Coventry University, CV 5FB, UK \\ ${ }^{[3]}$ Henry Moseley X-ray Imaging Facility, School of Materials, University of Manchester, M13 9PL, UK \\ ${ }^{[4]}$ Diamond Light Source, Harwell Oxford Campus, Didcot OX11 0DE, UK \\ ${ }^{[5]}$ Aerospace Research Institute, University of Manchester, M13 9PL, UK
}

\begin{abstract}
This paper develops a new fibre tracking algorithm to efficiently locate fibre centrelines (skeletons), from X-ray Computed Tomography (X-ray CT) images of carbon fibre reinforced polymer (CFRP), which are then used to generate micro-scale finite element models. Threedimensional images with 330nm voxel resolution of multidirectional [+45/90/-45/0] CFRP specimens were obtained by fast synchrotron X-ray CT scanning. Conventional image processing techniques, such as a combination of filters, delineation of plies, binarisation of images, and fibre identification by local maxima and ultimate eroding points, were tried first but found insufficient to produce continuous fibre centrelines for segmentation, especially in regions with highly congested fibres. The new algorithm uses a global overlapping stack filtering step followed by a local fibre tracking step. Both steps are based on the Bayesian inference theory. The new algorithm is found capable of efficiently define fibre centrelines for the generation of micro-scale finite element models with high fidelity.
\end{abstract}

Keywords: A. Carbon fibres; B. Microstructures; C. Finite element analysis (FEA); D. CT analysis

\section{INTRODUCTION}

$\mathrm{X}$-ray computed tomography (X-ray CT) is a technique being increasingly used to understand the relationship between the fibre architecture and the mechanical performance of fibre reinforced composites, owing to its non-destructive nature, and the capability of producing high-resolution

"Corresponding author: Prof Z Yang, ac1098@ coventry.ac.uk 
and clear visualisation of 3D internal structures of the material at nano- and micro-scales [1, 2]. This technique, integrated with in-situ loading rigs, allows the microstructural evolution inside the composites to be observed, for example, to track the onset of fibre fracture, delamination, transverse-ply cracking etc. [3-5]. X-ray CT image-based numerical models (most commonly the finite element (FE) method) have also been recently built to investigate the mechanical behaviour of some multi-phase materials, such as homogenisation of elastic properties of fibre reinforced concrete [6], damage evolution of carbon-carbon composites [7], and complicated nonlinear fracture in concrete [8,9]. The marriage of in-situ X-ray CT tests and numerical simulations is now emerging as a very promising tool for both validation of complicated numerical models and optimisation of internal structures of composite materials.

The approaches for generation of FE models from X-ray CT images can be categorised into two classes: (i) direct voxel (or pixel) to mesh mapping and (ii) phasic segmentation based meshing. In the first class, each voxel or pixel in the image is directly transformed into a finite element, and the resultant bi-material interfaces in the mesh can be further smoothed. The material constituents are distinguished based on their respective grayscale intensity values or CT numbers. Such approaches are widely reported, e.g., the direct voxel mapping methods [8, 10], the discrete Fourier transform approach [11, 12], and the enhanced volumetric marching cubes [13]. A problem of these approaches is that automatic meshing algorithms are difficult to apply and the element sizes are usually too uniform. In the second class of approaches, the X-ray CT image is segmented into different constituent phases based on the gray value thresholds for each phase. The bi-material interfaces or phasic boundaries can be naturally identified and smoothed if necessary. Generation of FE meshes is then conducted based on the segmented images. Examples of fibre reinforced composites include the combined segmentation and voxel-based fibre tracking algorithm [14], the simplified marching cubes method [15-17], the fibre contacts and orientation method [18, 19] and the intersecting fibre cluster method [20]. The main advantage of direct segmentation for fibre composites is that various $3 \mathrm{D}$ skeletonisation algorithms can be applied to extract fibre centrelines $[18,20]$ after segmentation, so that the fibres can be modelled as exact cylinders. Another advantage is that the segmented images can be automatically meshed with flexible control so that dense meshes can be used in stress concentration areas such as bi-material interfaces.

However, most of the above approaches are difficult to implement for fibre reinforced composites with highly congested fibres and/or touching fibres, before FE models can be built. The difficulties include finding exact segmentation boundaries, fibre tracking due to inherent X- 
ray $\mathrm{CT}$ noises and fibre discontinuities, and high computational cost due to large $\mathrm{X}$-ray $\mathrm{CT}$ datasets. The low image contrast between the polymer matrix and the thin fibres with thickness of a few microns may also lead to FE meshes with inaccurate material interfaces. New numerical reconstruction methods with advanced image filtering and stack conditioners have thus been developed for fibre tracking and noise reduction. For example, Czabaj and co-workers developed a template matching algorithm to determine the fibre centre points from X-ray CT images of unidirectional carbon fibre reinforced polymers (CFRP) [2, 21]. The fibre centre points are then converted into higher-order splines followed by sweeping non-overlapping $3 \mathrm{D}$ volumes along each spline. The method has not been tested on multidirectional fibre volumes.

This paper is aimed at efficiently building high-fidelity micro-scale CT image-based FE meshes for 3D, cross-plied, multidirectional fibre reinforced composites by developing a new fibre tracking algorithm to resolve some of the above issues. The images of a notched CFRP cylinder with [+45/90/-45/0] fibre orientation sequence are captured by fast synchrotron X-ray CT scanning with $330 \mathrm{~nm}$ voxel resolution. The images are then analysed and rendered in the commercial software Avizo (FEI/Avizo, 2016) and reconstructed using the developed algorithm implemented in the open-source code ImageJ [22]. After the fibre centrelines are identified by the new fibre tracking algorithm, which is based on the ultimate eroding points and the Bayesian inference theory, 3D micro-scale FE models are built in ABAQUS. The developed algorithm can also be used to characterise fibre waviness, tortuosity, orientation etc. and the FE models will be used to simulate 3D micro-scale damage and fracture evolution in CFRP in the future.

\section{IN-SITU SYNCHROTRON X-RAY CT EXPERIMENTS}

It is known that the images of CFRP obtained from conventional X-ray CT machines have relatively low phase-contrast, due to similar material densities between the carbon fibres and the epoxy resin. In this study, the $3^{\text {rd }}$ generation synchrotron radiation (beamline I13) at Diamond Light Source in Oxfordshire, UK with high brilliance is used to enhance phase contrast [23]. An in-house loading rig is designed and used for the in-situ uniaxial tensile test, as shown in Fig. 1a.

The investigated material is carbon fibre/epoxy resin cross-ply composite with a ply sequence [$45 / 90 /+45 / 0 /-45 / 90 /+45 / 0]_{\mathrm{s}}$. The manufacturer specification of the carbon fibre diameter is $5.2 \mu \mathrm{m}$. The matrix is pre-impregnated epoxy resin with a tensile strength of 50MPa. The average fibre volume fraction for individual unidirectional plies is $50 \%$. Figure $1 \mathrm{~b}$ illustrates a sample with a nominal thickness $1.8 \mathrm{~mm}$, prepared using a diamond water blade and notched on two sides using a carbide blade of $0.3 \mathrm{~mm}$ thickness. Thin steel metal end tabs are glued to the 
specimen using a bi-component epoxy adhesive so that it could be loaded in-situ on the X-ray CT stage.

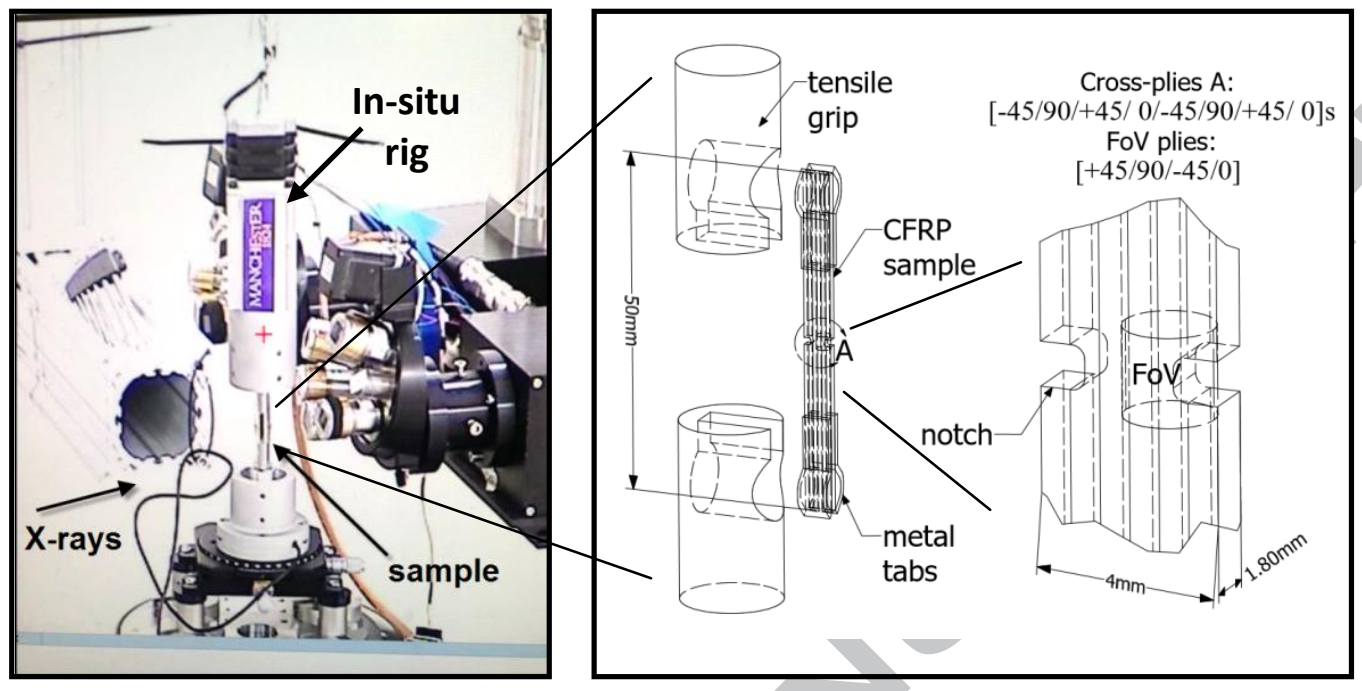

Fig. 1: In-situ X-ray CT tensile test of a CFRP specimen using an in-house loading rig

The gap of the undulator (a $2 \mathrm{~m}$ long U22) was set to $5.5 \mathrm{~mm}$ for increased flux. Intense radiation over an extended energy bandwidth was used to enhance phase contrast and speed up image acquisition. The energy bandwidth was carefully tuned by $3 \mathrm{~mm}$ Al filters inserted into the beam path to attenuate soft $\mathrm{X}$-rays below $18 \mathrm{keV}$. This complemented the cut-off of the platinum mirror stripe at $25 \mathrm{keV}$, allowing good penetration of the sample whilst maintaining sample visibility. This mirror was slightly focussed to collimate in the horizontal direction and to counteract the large horizontal divergence of the source, giving around $7 \times 10^{13}$ photons per second into the field of view $(\mathrm{FoV})$ of $712 \mu \mathrm{m}$ (height) $\times 840 \mu \mathrm{m}$ (diameter). The beam was propagated about $30 \mathrm{~mm}$ before detection, making use of the edge enhancement from the inline phase-contrast to pick up individual carbon fibres in the matrix.

A PCO edge 5.5 CMOS detector based microscope system with 14bit dynamic range was used. The system images the light generated by the X-rays via a GGG:Eu scintillator with a 10x magnification lens. A $2 \times$ tube lens in the microscope led to a total $20 \times$ magnification factor of the scintillated images, providing an effective pixel size of $330 \mathrm{~nm}$ at the CMOS detector. The fast readout of this detection system (up to $100 \mathrm{~Hz}$ ), combined with the high flux from the beamline, allowed tomograms to be collected in the fly-scan mode at $0.2 \mathrm{~s}$ per exposure. More detailed information about the instrumentation can be found in [24]. 
Reconstruction of 3D images from the X-ray CT attenuation data is conducted using the wellknown filtered back projection approach [25]. The dataset consists of a stack of 2160 slices each comprising $2560 \times 2560$ pixels. Fig. 2a shows the rendered FoV in Avizo using the volrenGreen tool. Fig. $2 \mathrm{~b}$ shows the fibres in a small cube with length $50 \mu \mathrm{m}$. It can be seen that the fibres are blurred, discontinuous and not clearly defined. A crack initiating from the notch, captured at the peak load (about $600 \mathrm{~N}$ ) from an in-situ X-ray CT test under quasi-static uniaxial tension, is also clearly visible.

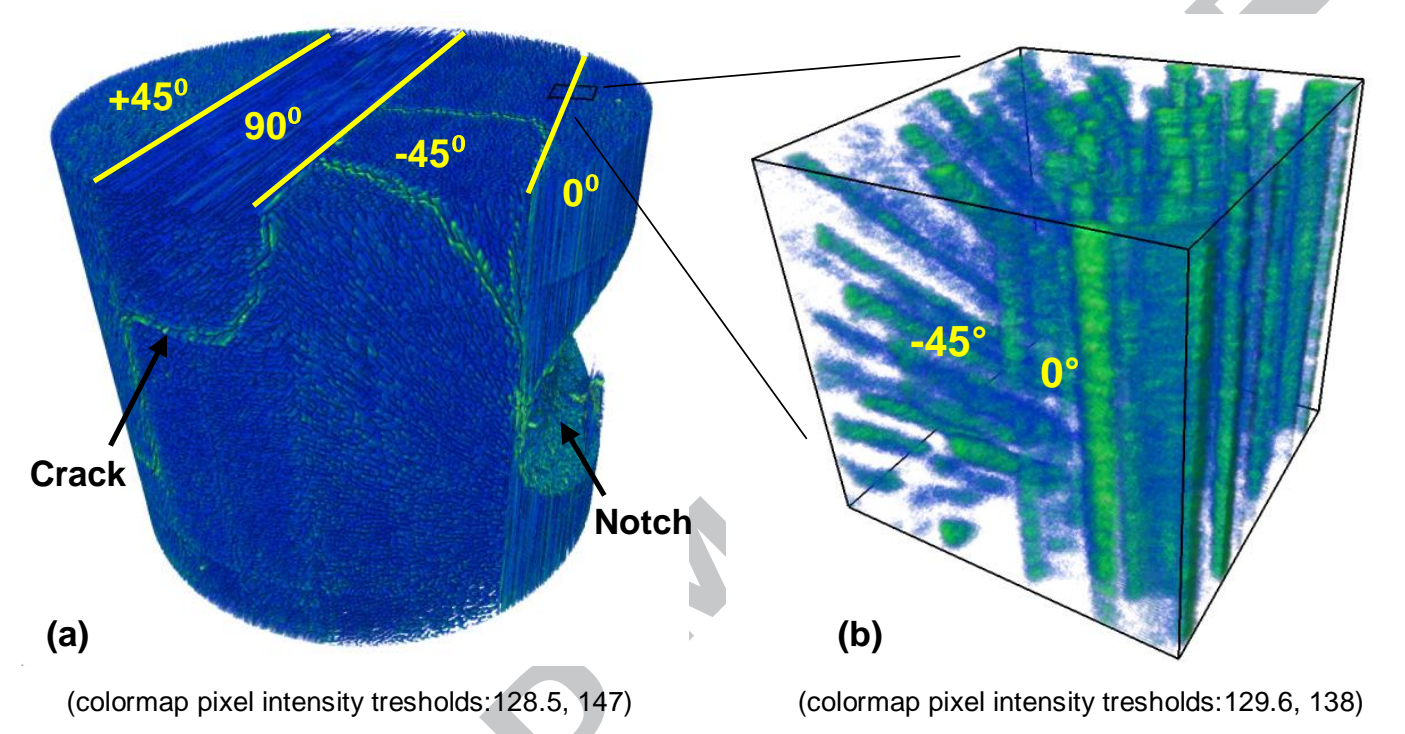

Fig. 2: X-ray CT volume render of a multidirectional CFRP specimen

\section{IMAGE-BASED MODEL GENERATION}

Fig. 3 shows the main steps to extract the CFRP geometry from X-ray CT image slices and generate FE meshes. Firstly, effective filters were identified and applied to the stack of X-ray CT images to remove noises and facilitate fibre identification. The 3D dataset was then delineated into plies according to fibre orientations. The fibre centres were then identified using the ultimate eroding point approach. Image binarisation was also tried using the traditional watershed splitting and skeletonisation techniques. The conventional approaches failed to obtain continuous fibre centrelines. Therefore, a new algorithm for fibre tracking with two steps using stack conditioning incorporating the Bayesian inference was developed. The obtained fibre centrelines were finally processed with consideration to manufacturing practice of CFRP, before they are used to generate FE meshes in ABAQUS assuming a constant fibre diameter. 


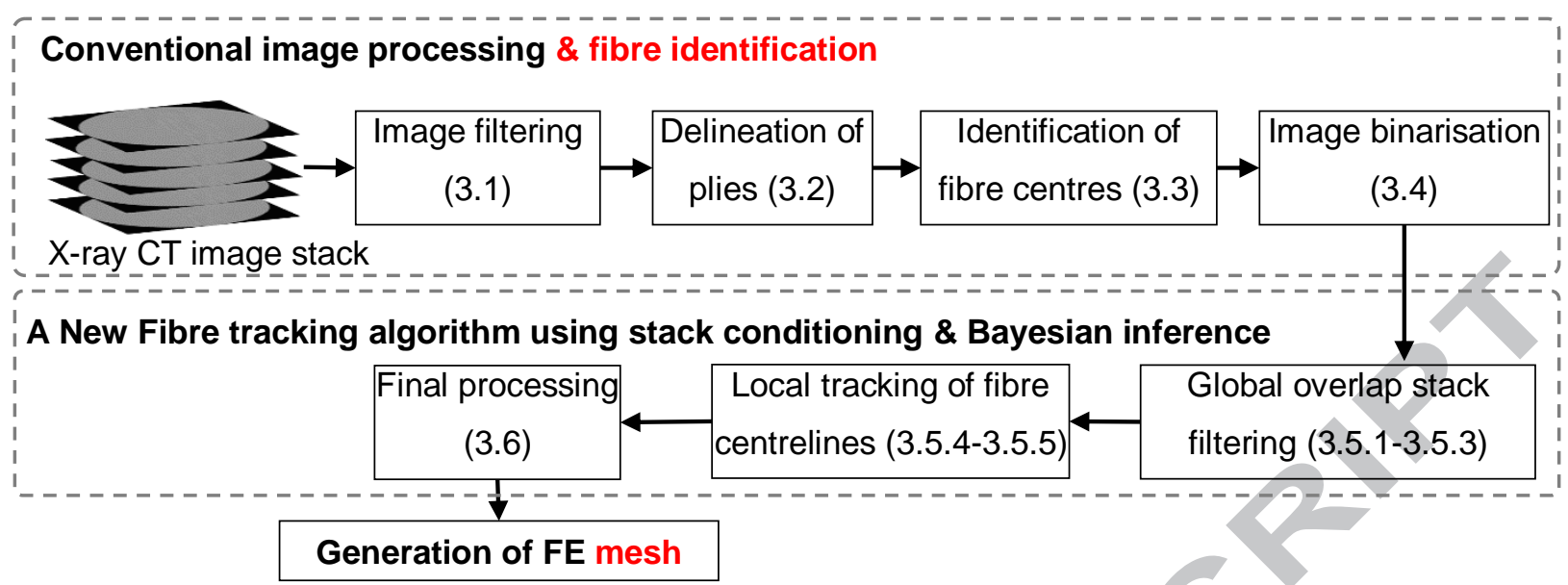

Fig. 3: Illustration of the image-based model extraction framework from X-ray CT to FE meshes

\subsection{Images filtering}

Fig. 4 shows half of a typical raw X-ray CT image slice. The low phase-contrast and pixel oversaturation can be seen. Also shown in Fig. 4 is a small region of interest (RoI) after different combinations of filters (Filter-A to Filter-E as detailed in Table 1) were applied. These filtering operations were implemented using the JavaScript library in ImageJ. None of the common filters from Filter- $A$ to Filter- $D$ alone was able to enhance the detectability and shape of congested fibres as they used fixed stencil and gradient based kernels.

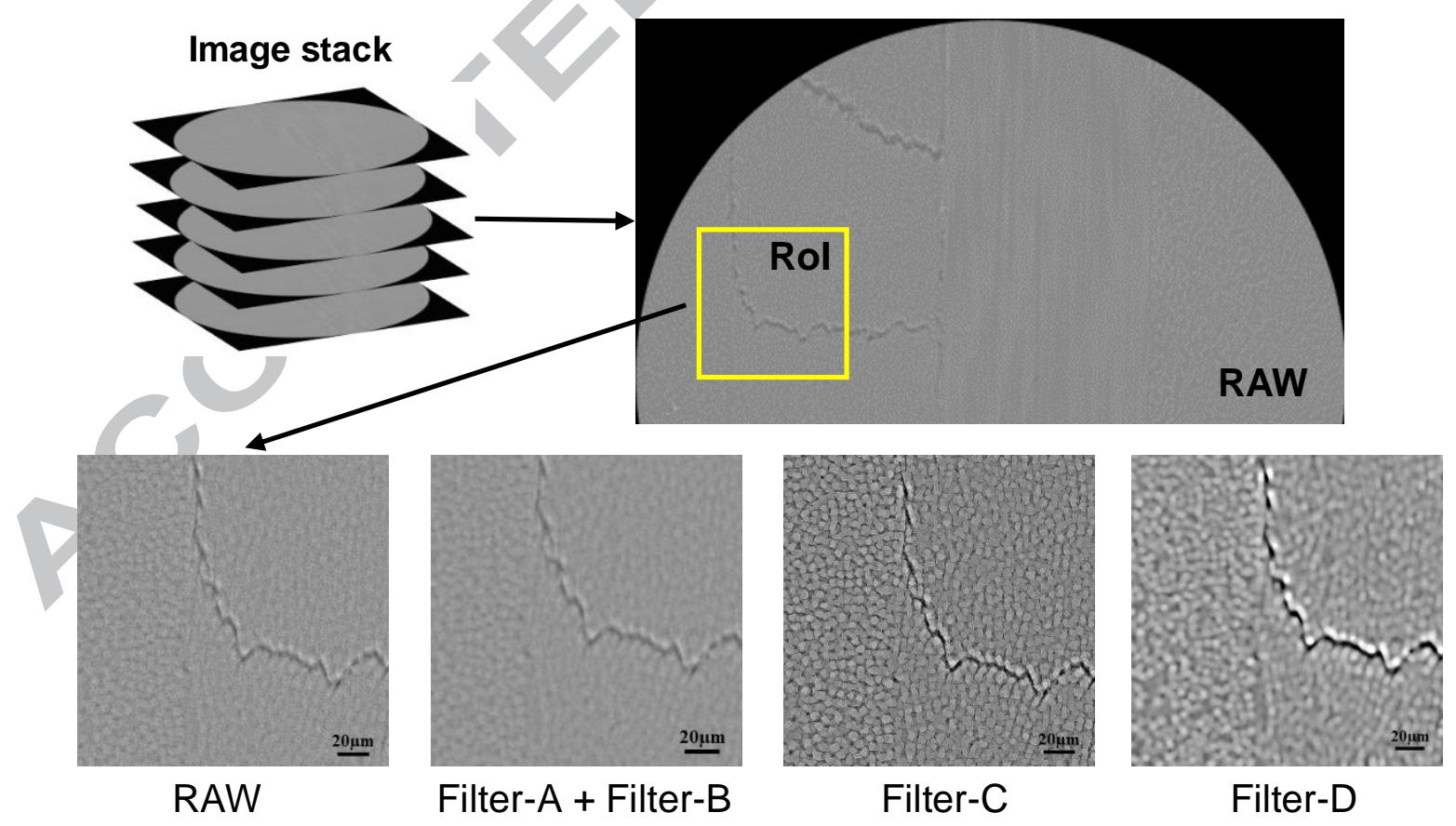

Fig. 4: X-ray CT image filtering by different filter combinations in Table 1. 
Table 1: Image filters for X-ray CT datasets.

\begin{tabular}{|l|l|l|}
\hline Reference & Image Filter Combinations \& Order & Intended use \\
\hline Filter- $A$ & Mean 2pixels - Smooth & $\begin{array}{l}\text { De-noising, intensity } \\
\text { smoothening. }\end{array}$ \\
\hline Filter - $B$ & $\begin{array}{l}\text { Mean 2pixels twice - Smooth - Unsharp mask (radius: 7 pixels, } \\
\text { mask weight: 0.2) }\end{array}$ & $\begin{array}{l}\text { Phase contrast, Edge } \\
\text { enhancing, Interface } \\
\text { threshold. }\end{array}$ \\
\hline Filter-C & $\begin{array}{l}\text { Smooth - Mean 2px - Min maxima \& region growth 2.5pixels - } \\
\text { Max maxima \& region growth 3pixels }\end{array}$ & $\begin{array}{l}\text { Pragmatic fibre } \\
\text { centreline network } \\
\text { search. }\end{array}$ \\
\hline Filter-D & $\begin{array}{l}\text { Smooth - Median 7pixels - Min maxima 3pixels - Max maxima } \\
\text { 2.5pixels - Unsharp mask (radius: 7 pixels, mask weight: 0.2), } \\
\text { Enhance contrast (saturated pixels: 0.01\% /normalise) }\end{array}$ & $\begin{array}{l}\text { High fidelity fibre } \\
\text { centres \& fibre } \\
\text { identification. }\end{array}$ \\
\hline Filter-E & $\begin{array}{l}\text { Smooth - Mean 2px - Kernel convolution - Max maxima 2.5 } \\
\text { pixels - local maxima search for fibre identification. }\end{array}$ & $\begin{array}{l}\text { Fidelity fibre centroids \& } \\
\text { fast processing. }\end{array}$ \\
\hline
\end{tabular}

The identified image filter strategy with best effect is a combination of a series of filters (Filter$E$ in Table 1). A fixed size kernel convolves all the pixels contained within the image slices. The convolution uses a continuous stencil pattern (pixel-wise) and the new images are obtained through multiplication and weight summation of the $2 \mathrm{D}$ matrices overlaid as demonstrated by Eq. 1:

$$
\operatorname{conv}(f, k)=f_{x y} \otimes k_{x y}=\sum_{e_{w}=-\frac{w}{2}}^{\frac{w}{2}} \sum_{e_{h}=-\frac{h}{2}}^{\frac{h}{2}} f\left[\mathrm{x}+e_{w}\right]\left[\mathrm{y}+e_{h}\right] * k_{x y}\left[w / 2-e_{w}\right]\left[\mathrm{h} / 2-e_{h}\right]
$$

Eq. 1

where $f_{x y}$ are the pixel intensities from the image overlay and $k_{x y}$ is the processing kernel of size $[(w+1)(h+1)]$ with the square size kernel property $w=h$. This approach has been widely used in the image processing, especially for edge detection and target tracking [26, 27]. 2D convolution operations are typically implemented using vector operations such as the one-dimensional convolution approach. For example, Habibi et al. [28] described a decomposition strategy using two consecutive vertical and horizontal convolution steps for a 3 by 3 kernel size. In this study the size of kernel and convolution factors are semi-empirically estimated using Eq. 2 that 
represents the general probability density kernel [29]. This is fitted iteratively by trial and error adjusting the sample values $x_{i}$.

$$
k_{x y}=k_{e}(x)=\frac{1}{n h} \sum_{i=1}^{n} k_{s}\left(\frac{x-x_{i}}{h}\right), \quad-\infty<x<\infty
$$

where $n$ is the kernel size, $k_{\mathrm{s}}$ the kernel smoothing function and $h$ the bandwidth $(h=1)$. A similar model can be found in [30]. Fig. 5 shows the parametric estimation of the convolution factors based on a few popular smoothing functions $k_{s}$ used in Eq. 2.

The step function $k_{e}$ illustrated in Fig. 5 is used to estimate the convolution factors $k_{x y}$ in Eq. 1 . Because the average fibre diameter is $5.2 \mu \mathrm{m}$ and the fibres are highly congested in the X-ray CT dataset, the size of the convolution and local filtering kernels in Eq. 1 and Eq. 2 are set at $5 \times 5$ pixels, which means the convolution factors taken from the step function in Fig. 5 are located between -2 and 2 .
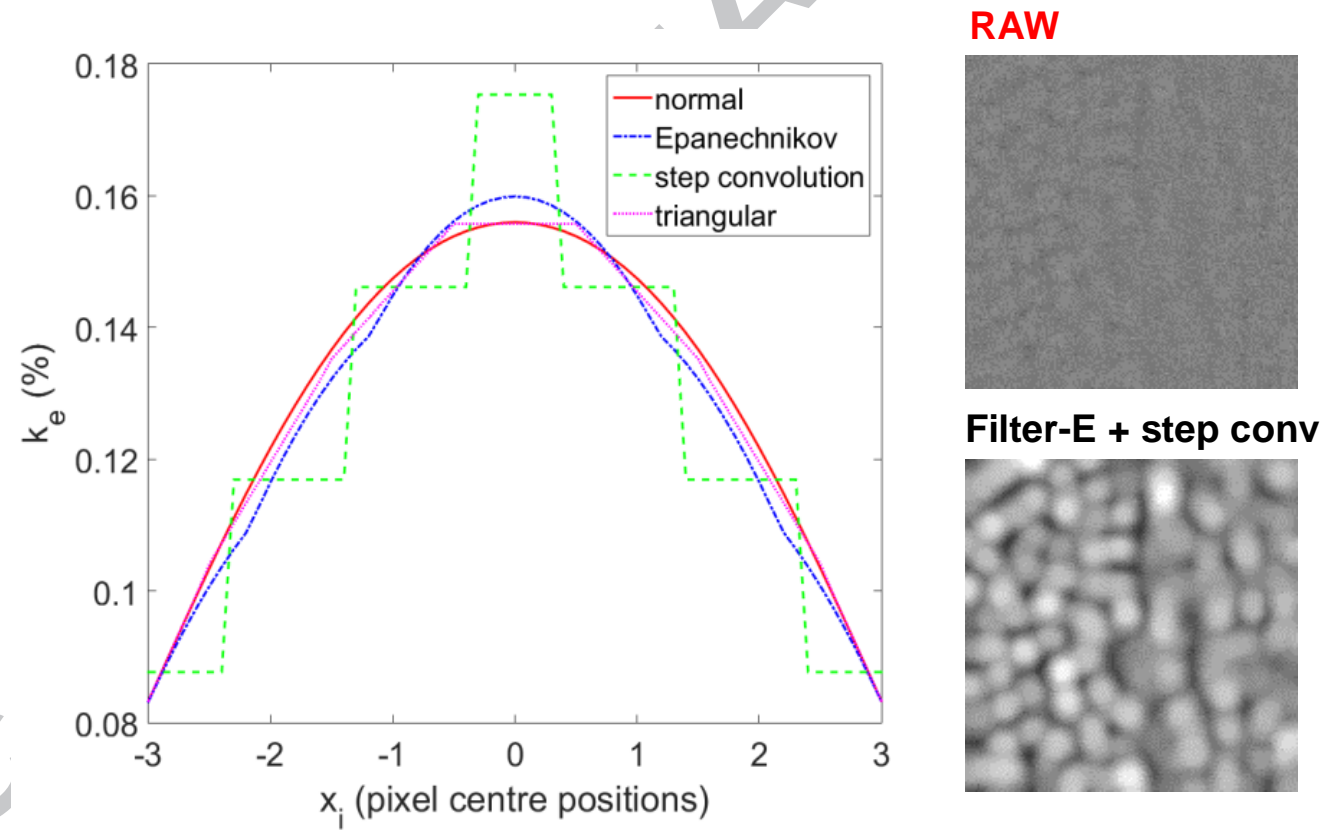

Fig. 5: Estimation of the convolution factors based on different smoothing functions; the insets typify the effect of the combined filter Filter- $E$ and convolution using the step function estimates at the boundary between $0^{\circ}$ to $-45^{\circ}$ plies.

\subsection{Volume delineation to different plies}

After image filtering and before fibre identification, it is necessary to split or delineate the X-ray CT volume into plies. The delineation was carried out using a changing polygon algorithm with linear pitch between the corresponding extraction corner points which are located within the first 
and last slices of the X-ray CT dataset. As the transverse eroding basin approach ultimately leads to a single point identification [31], the current fibre identification method requires transverse scans with fibre orientations $<50^{\circ}$. Consequently, the FoV was separated into two sub-domains, one with fibres oriented at $<50^{\circ}\left(0^{\circ}\right.$ and $+/-45^{\circ}$ plies in Fig. 6a) and another with fibre orientation of $90^{\circ}$ (Fig. 6b). The two sub-domains were then combined to form a single yolume geometry (in Fig. 6f).

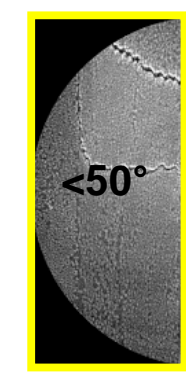

(a)
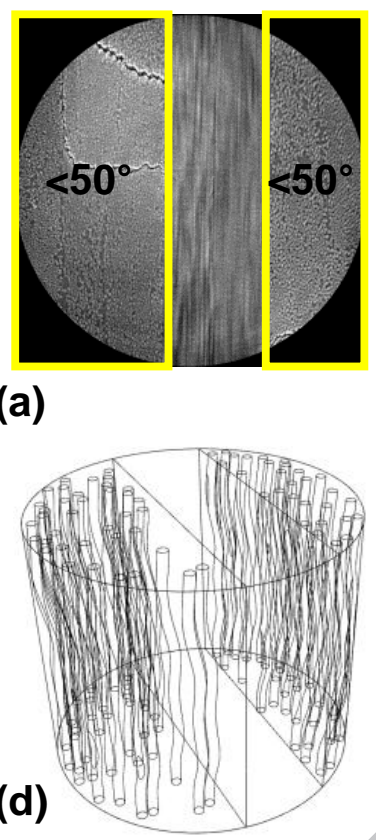

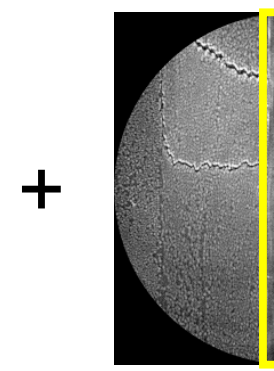

(b)

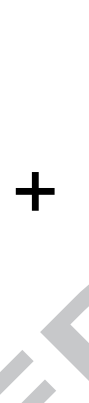

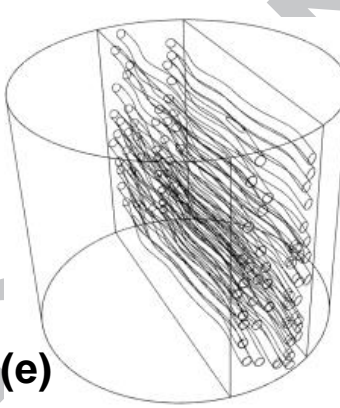

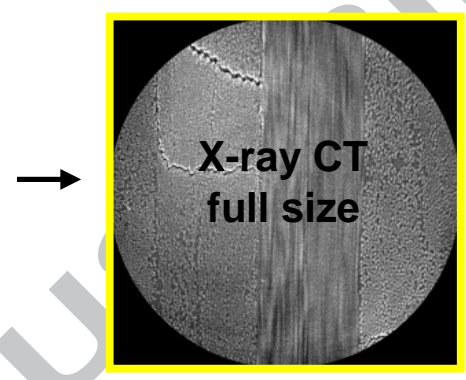

(c)

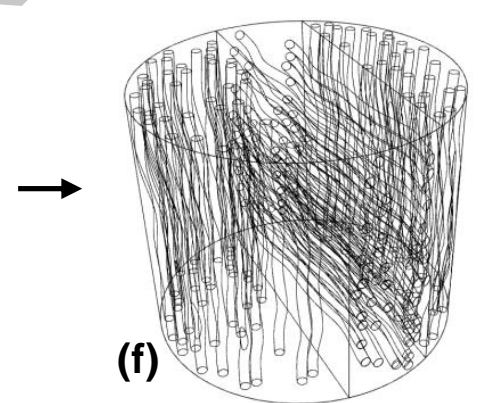

Fig. 6: Illustration of volume delineation based on fibre orientations

\subsection{Identification of fibre centres}

The well-established local maxima finding algorithm in ImageJ was applied to the filtered image slices to identify ultimate eroding points (UEP) as fibre centres [31]. The UEPs are obtained using a procedure combining watershed delimitation basins and eroding Euclidean maps. The algorithm is able to retrieve the fibre centre points from the $330 \mathrm{~nm}$ image resolution datasets in this study with relatively low errors, as can be seen from the tortuosity of fibre volumes shown in Fig. 7. However, Fig. $7 \mathrm{~b}$ shows that due to misidentification of UEPs of some fibres, fibre discontinuities still arose, particularly in inclined ply regions with highly congested fibres, where the identification of separate Euclidean maps became difficult. 
(a) Volume render

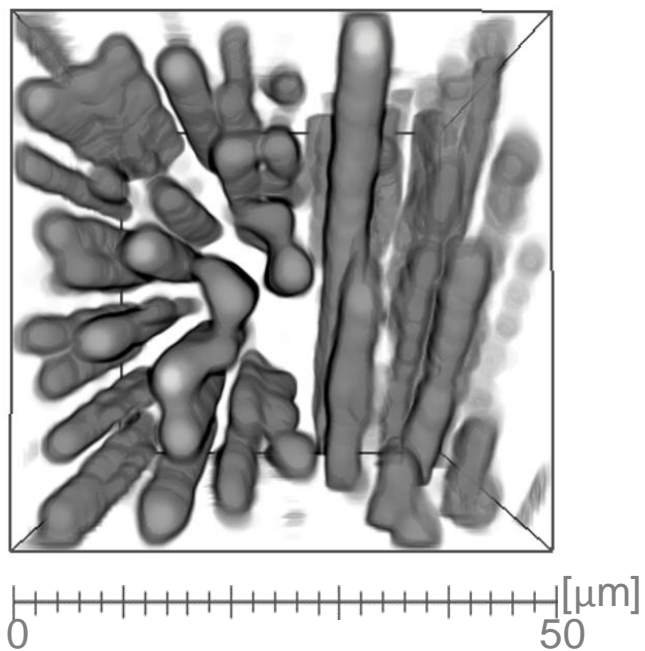

(b) Identified fibre UEPs

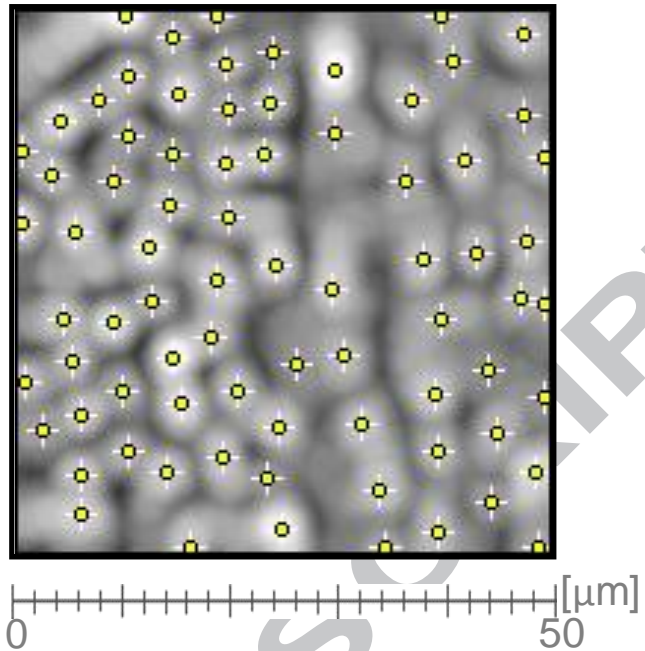

Fig. 7: Identification of the fibre centroids after image filtering in a RoI at the $0 \%-45^{\circ}$ ply boundary (bounding box corner=255,1400,0 \& 152 voxels): (a) volume render after image filtering and (b) identified UEPs in top image slice

\subsection{Image binarisation}

Further to the image filtering and the identification of UEPs in previous sections (3.1 and 3.3), the 2D image slices were converted into binary images (Fig. 8a) by growing uniform intensity fibre domains of equal diameters centred on the UEPs. The well-known water basins approach was used to separate touching fibres when necessary. A volume render based on the binary image slices is shown in Fig. 8b, from which either direct segmentation or skeletonisation to retrieve the fibre centrelines by volume shrinking can be carried out. However, it was found that this reconstruction method was not only computationally expensive due to the large number of fibres, it could also result in imprecise boundaries and discontinuous fibres in volume renders (see Fig. 8b). 

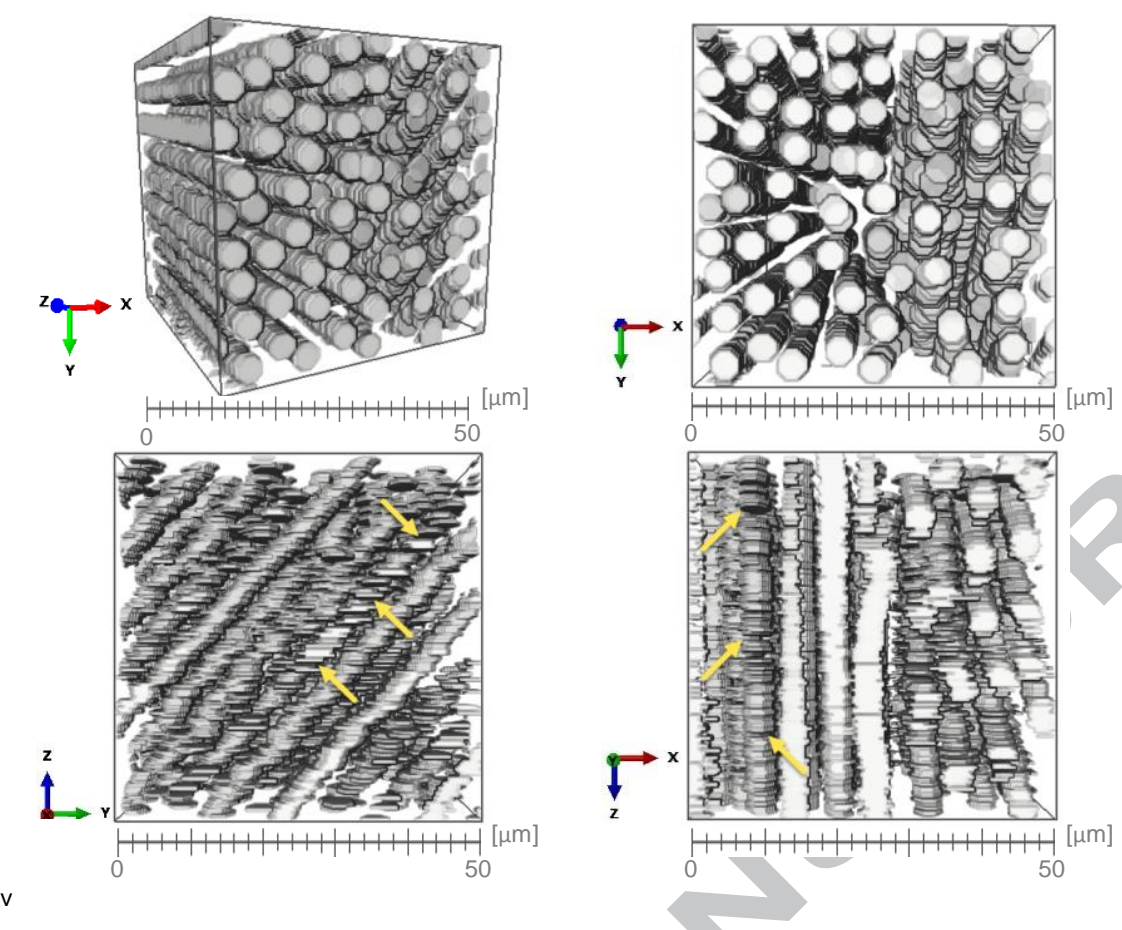

Fig. 8: Fibre tracking from binary images in a RoI at the $0^{\circ} /-45^{\circ}$ ply boundary (the fibre discontinuities are highlighted by arrows).

\subsection{New fibre tracking algorithm with stack conditioning}

To acquire more continuous fibre centrelines with lower computational cost than the conventional image processing techniques tried and discussed above, a new fibre tracking algorithm was developed. It consists of two steps: a global stack overlap filtering and a local fibre centreline tracking, both employing the Bayesian inference, as presented below.

\subsubsection{Global overlapping stack filtering}

After the identification of fibre centre points (UEPs) and the image binarisation, an overlapping discretisation of the continuous slices was conducted to find the best matching slices. This step is necessary to test continuity of the fibres and to transpose data in the best possible filtered stack. It can also lower the computational cost compared with tracking fibres in $3 \mathrm{D}$ within the full stack.

The idea of stack overlap filtering is illustrated in Fig. 9. The total stack domain $D$ comprises 2160 CT image slices. The images within the kernel stack domain $d$ are iteratively correlated in pairs covering all combinations with respect to the stack centre. A first global filter can be then applied according to the degree of matching between all image pairs. 


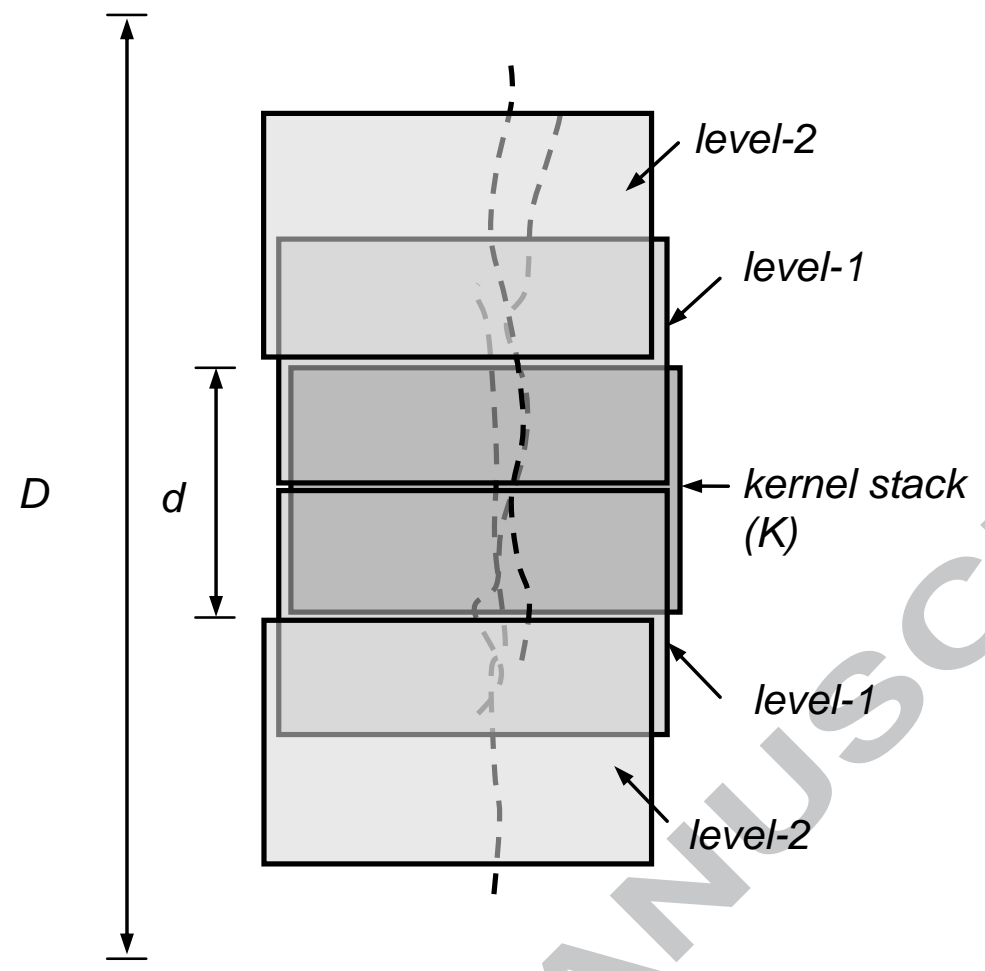

Fig. 9: Bayesian inference domains for fibre tracking: kernel stack domain $(d)$ and stack overlap $(d / 2)$.

\subsubsection{Estimation of image matching and fibre shifts}

The matching or similarity of two images with pixel intensity distribution function $f$ and $g$ can be estimated by their cross-covariance:

$$
\operatorname{cov}(f, g)=E\left[(f(x, y)-\bar{f})(g(x+i, y+j)-\bar{g})^{T}\right]
$$

where $\bar{f}$ and $\bar{g}$ are the average pixel intensity values, and $i$ and $j$ are the integer image shift. The mathematical expectation of pixel intensity of an image $f$ with $m \times n$ pixels is

$$
\left.E(f-\bar{f})=\sum_{m, n}[f(x, y)-\bar{f})\right]
$$

Note that the variance of an image $f$ can be written as:

$$
\operatorname{cov}(f, f)=E(f-\bar{f})^{2}=\sigma^{2}(f)
$$

The Normalised Correlation Coefficient (NCC) is defined as: 


$$
C_{N C C}=\operatorname{corr}(f, g)=\frac{\operatorname{cov}(f, g)}{\operatorname{sqr}\left(E(f, \bar{f})^{2} E(g, \bar{g})^{2}\right)}
$$

The NCC index measures the degree of similarity or matching and ranges between 0 and 1 . Furthermore, a global integer shift can be obtained by maximising the NCC index. Similarly, by maximising the NCC index a local integer fibre shift can be found by using smaller image masks. If needed, a sub-pixel shift can also be found by applying the local quadratic fitting around the peak value $(\Delta x, \Delta y)$ of the correlation results:

$$
\begin{aligned}
& \delta x=\frac{\operatorname{corr}(\Delta x-1, \Delta y)-\operatorname{corr}(\Delta x+1, \Delta y)}{2[\operatorname{corr}(\Delta x-1, \Delta y)+\operatorname{corr}(\Delta x+1, \Delta y)-2 \operatorname{corr}(\Delta x, \Delta y)]} \\
& \delta y=\frac{\operatorname{corr}(\Delta x, \Delta y-1)-\operatorname{corr}(\Delta x, \Delta y+1)}{2[\operatorname{corr}(\Delta x, \Delta y-1)+\operatorname{corr}(\Delta x, \Delta y+1)-2 \operatorname{corr}(\Delta x, \Delta y)]}
\end{aligned}
$$

where $\delta x$ and $\delta y$ range between -0.5 and +0.5 pixels. For completeness, the total image shift can be estimated by the summation of results in Eq. 7 as an integer. It is used to estimate the total metric threshold for fibre tracking in inclined plies, and the threshold is further used by the local Bayesian inference model to track individual fibres (see Sections 3.5.4 and 3.5.5).

\subsubsection{Global Bayesian inference for optimal stack width}

To reduce the large number of slices from an X-ray CT dataset, the slices within a kernel were correlated in pairs with respect to the overlapping stack centre. The filtered slices can be selected by maximising the NCC index over each kernel domain (meaning the selected slice contains most similar pixel patterns out of the total number of pairs). Instead of using pixels, the slices can be simplified as separate sets of independent fibre UEPs. Therefore, the filter iterated over a prescribed number of half overlapping kernel stacks as shown in Fig. 9. The overlapping stacks were thus divided into two equal subdomains $\left(K_{d}=\mathbf{K}+\mathbf{N}\right)$. For all slices contained in half of a kernel stack $(\mathbf{K})$, the probability field $\mathrm{P}(K)$ was defined as the total number of UEPs contained within the slice $K$. The neighbouring slices in $\mathbf{N}$ were also defined in a similar manner via prior probability $P(\mathbf{N} \mid K)$ that contained the corresponding UEPs. By iterating over the slices in $\mathbf{K}$, the slice UEP filtering from similar probabilities $P(K \mid \mathbf{N})$ could be found by maximising the following Bayesian equation: 


$$
P(K \mid \mathbf{N})=\frac{P(\mathbf{N} \mid K)}{\sum P(\mathbf{N} \mid \bar{K}) P(\bar{K})} \cdot P(K)
$$

where $\mathbf{N}=\left\{\mathrm{n}_{1}, \mathrm{n}_{2}, \mathrm{n}_{3}, \ldots, \mathrm{n}_{k}\right\}$ represent the UEP sets from the corresponding slices within half the kernel width (i.e. $k=d / 2$ ). In Eq. $8 \bar{K}$ denotes the slice that contains the average number of UEPs over stack K. $P(\mathbf{N} \mid K)$ represents the probability of all slices within kernel $\mathrm{K}_{\mathrm{d}}$.

This method can be applied when the kernel stack is relatively narrow and the overlapping region captures the inclined fibre plies as illustrated in Fig. 10. In contrast, the number of correlated fibres is relatively large for the aligned fibre plies as shown by the correlated images in Fig. 10d and e (shown by the left hand ply points). Because the method correlates all slices within a given stack width, the fibres that do not cross this full-width are not detected. To solve this problem, the method was calibrated using a sensitivity study as explained below. Results in Table 2 indicate that a kernel width of 8 slices can be used that reduces the computational stack 4 times.
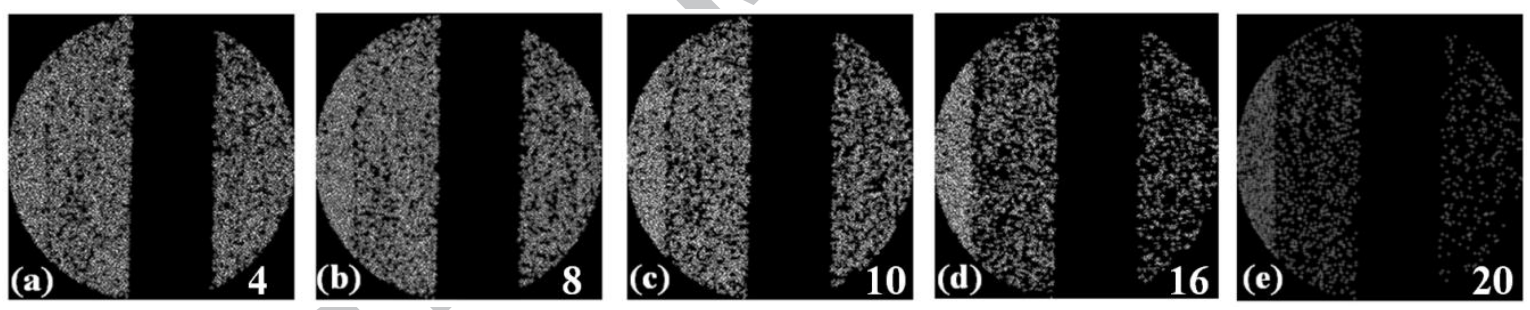

Fig. 10: Sensitivity of fibre tracking UEPs with different Bayesian overlapped stack widths, using the parameters in Table 2 .

In order to capture fibre continuity, the kernel width $d$ should be sufficiently large to avoid misidentified UEP centres, but also reasonably small to reduce the computational time. The optimal kernel width is determined by inspection of fibre discontinuity visually in the volume render in Avizo, for example, Fig. 7. In this paper, it was found that the method could be applied to kernel widths equal to half the fibre diameter, namely, 2.6 $\mu \mathrm{m}$, as demonstrated in Fig. 10b and the optimum overlapping distance was found to be 4 slices. 
Table 2: Bayesian overlapped stack width sensitivity study for the inclined plies

\begin{tabular}{|c|c|c|c|c|}
\hline $\begin{array}{l}\text { Kernel width } d \text { in } \\
\text { number of slices }\end{array}$ & $\begin{array}{l}\text { Overlapping distance in } \\
\text { number of slices }\end{array}$ & $\begin{array}{l}\text { Initial number of } \\
\text { fibre UEPs }\end{array}$ & $\begin{array}{l}\text { Matched number } \\
\text { of fibres }\end{array}$ & $\begin{array}{l}\text { Image reference } \\
\text { in Fig. } 10\end{array}$ \\
\hline 4 & 2 & \multirow{5}{*}{6198} & 4847 & (a) \\
\hline 8 & 4 & & 4497 & \\
\hline 10 & 5 & & 4267 & (c) \\
\hline 16 & 8 & & 3017 & (d) \\
\hline 20 & 10 & & 2029 & (e) \\
\hline
\end{tabular}

\subsubsection{Local Bayesian inference for tracking individual fibres}

This local tracking step searches only within the filtered slices for individual fibre coordinates to ensure fibre continuity.

The general Bayesian inference theory was tailored to map the identified fibres in a kernel slice to the globally filtered neighbouring slices as follows:

$$
P(K \mid N)=\frac{P(N \mid K)}{\sum P(N \mid \bar{K}) P(\bar{K})} \cdot P(K)
$$

where $N$ are the UEP fibre centres coming from the neighbouring/overlapping domains with unknown probability $P(N \mid K)$ and $K$ are the UEPs from the searching slice, that are updated from slice to slice. The fibre UEPs from kernel slice $K$ here, are seen as events representing the total number of identified fibres in the slice. The probability $P(K \mid N)$ is therefore stored in a vector defined over a slice that can be also unequal to the neighbouring slice after the global filter due to errors in the identification process (especially in inclined fibre plies).

The denominator $P(N \mid \bar{K})$ represents a local conditional probability of a perpendicularly projected point that is the degree of belief of each fibre in $N$ to match its projection UEP point $\bar{K}$ versus the probability of the same point, i.e. $P(\bar{K})$. However, the denominator in Eq. 9 plays a minor role and can be also dropped or used as a scaling factor for normalisation and calibration purposes. 
The numerator in Eq. 9 is the probability $P(N \mid K)$ calculated using the asymmetric power law metric in Eq. 8, or can be directly limited by a prescribed fibre shift distance. When the power law metric is used to map and threshold the matching fibre coordinates, the probability can be expressed as follows:

$$
P(N \mid K)=x y e^{p}+y e^{q}
$$

where $(\mathrm{x}, \mathrm{y})$ are the coordinates of a fibre UEP and $(p, q)$ are two arbitrary power law indices with the asymmetric property $(p \neq q ; p, q<1)$. In this case, an initial threshold of 10 pixels is approximated as the equivalent to a maximum inclination angle of approximately $50^{\circ}$. The probability is then substituted back into Eq. 9, which is updated iteratively until processing of the filtered stack. Alternatively, a fibre shift distance can also be used. This shift can be estimated by maximising the NCC index in Eq. 6 or by calculating the distance between each two consecutive fibres. For more accurate geometric estimates, Eq. 7 can be used to quantify the fibre shift with sub-pixel precision. In this paper, the threshold distance is 8 pixels, namely, approximately half the fibre diameter.

\subsubsection{Calibration of local inference model}

The local Bayesian model discussed in Section 3.5.4 was calibrated using a series of restrictive fibre shift trials. These were implemented by an inverse exponential fitting to find the adaptive threshold coefficients using Fig. 10. Fig. 11 shows two such cases used to estimate the threshold values based on the two corresponding shifted probability distributions.
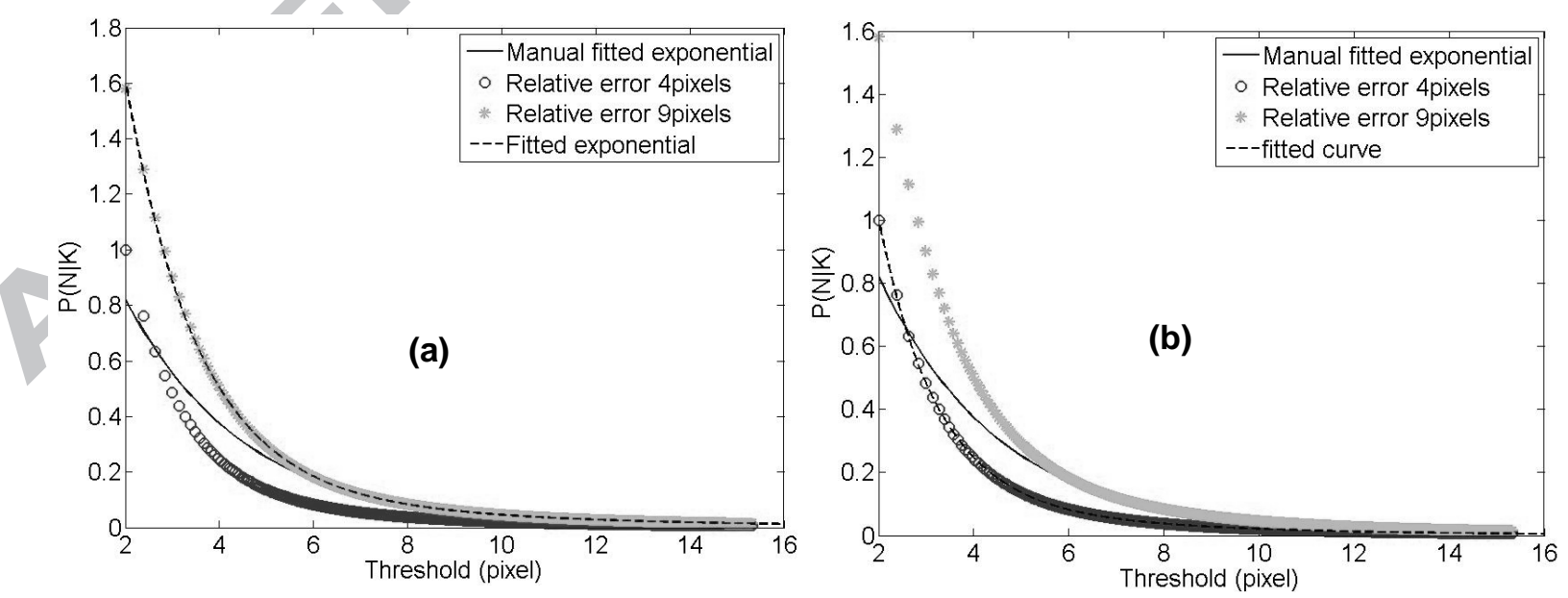

Fig. 11: Calibration of the local Bayesian tracking approach using

Eq. 10 by inverse fitting for: (a) tolerant fibre shifts equivalent to less than 9 pixels and (b) restrictive fibre shifts to less than 4 pixels (the power law factors are: $p=0.2, q=0.3$ ) 
One may note that [2] proposed a similar fibre tracking algorithm using the Kalman filtering. This algorithm also has two steps: a linear extrapolation of the fibre centre points is done first as a predictor, followed by a corrector minimising the local mean square error based on the adjacent slices. However, this algorithm follows a bottom-up approach and may not capture the continuity of inclined fibres if some fibre centroids are misidentified.

\subsection{Final processing of identified fibre centrelines}

The obtained data of fibre centrelines were further processed to consider the effects of real manufacturing process of CFRP, including: (1) removal of fibre centrelines with unrealistically short length, for example, less than the fibre diameter; and (2) splitting of fibre centrelines into segments. This operation is activated when the distance of two adjacent UEPs on a centreline is more than a predetermined gap distance, for example, $30 \mu \mathrm{m}$ in this study. This case may represent two fibres in reality but be interpreted as only one fibre by the above fibre tracking algorithm. The final fibre centrelines of the small region in Fig. $2 b$ are shown in Fig. 12. It can be seen that the fibre centrelines are now clearly defined.

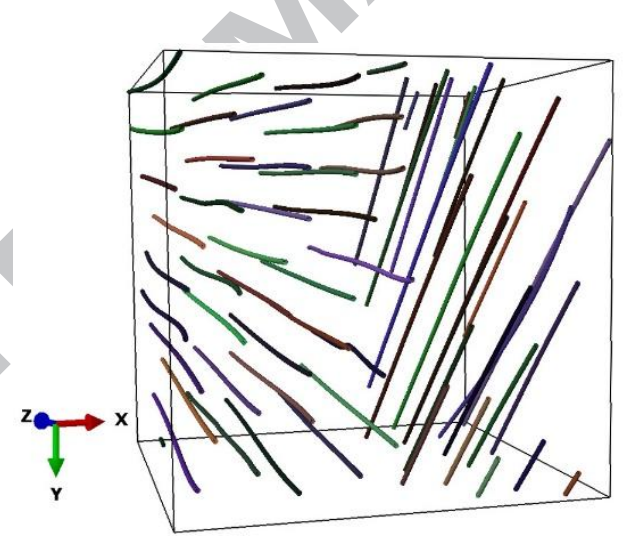

Fig. 12: Final extracted fibre centrelines of Fig. 2b (75 fibres).

\subsection{Geometry reconstruction and mesh generation}

The extracted fibre centrelines (modelled by third-order splines) in each ply were then integrated into a single domain of FoV as shown in Fig. 13, followed by image-based mesh generation. There are 19,954 fibres in total in Fig. 13c. 

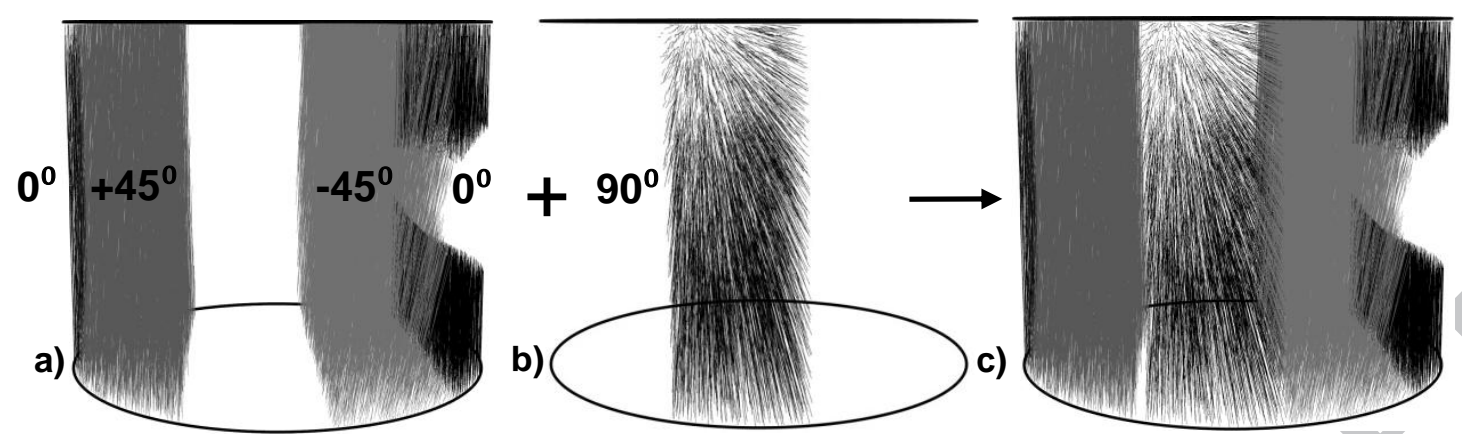

Fig. 13: Full-size X-ray CT reconstructed fibre centrelines (19,954 fibres).

It is known that the FE mesh size for accurate modelling of composite materials should be at

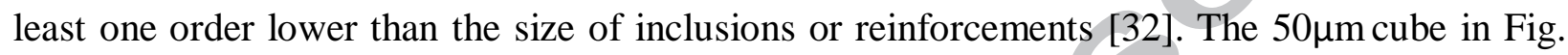
12 was converted into a FE mesh shown in Fig. 14 using the free meshing option in ABAQUS. The seed of element size was $0.667 \mu \mathrm{m}$ (note the fibre diameter $5.2 \mu \mathrm{m}$ ) and the mesh was generated in approximately 2 minutes. This mesh contained 75 fibres and about 2 million C3D4 elements. Converting the full FoV with 19,954 fibres would lead to a gigantic number (in billions) of finite elements which even supercomputers may struggle to model. This makes multiscale modelling necessary. However, 3D multi-scale modelling of quasi-brittle composites like CFRP and concrete is still largely limited to predictions of homogenised elastic properties and much research is still needed to develop more robust multi-scale methods for complicated damage and fracture evolution [32-34]. To this end, the in-situ X-ray CT tests undertaken in this study will prove very valuable in both providing high-fidelity initial FE models and also validating them by direct comparison of the simulated results with the CT images under progressive loadings [8-9]. 

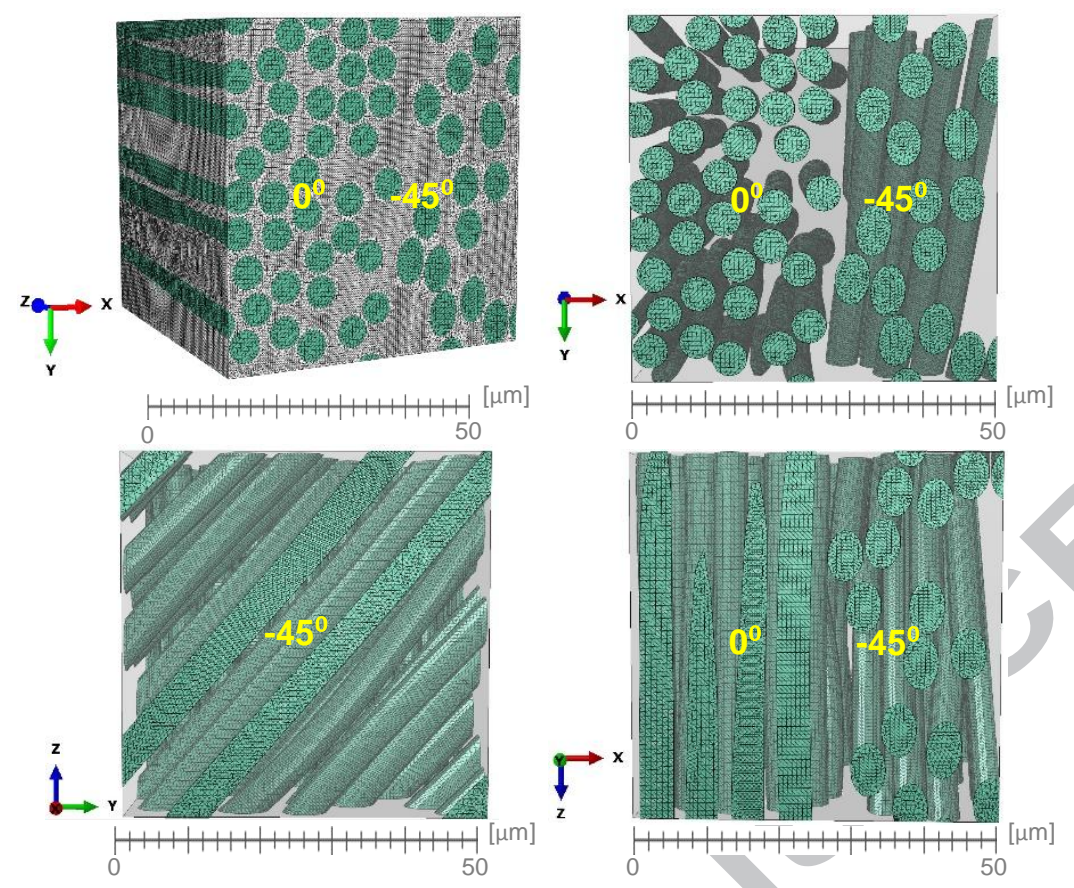

Fig. 14: X-ray CT image-based FE model

\section{CONCLUSIONS}

This paper has presented the development of a set of image processing and segmentation algorithms to efficiently identify fibre centreline (skeletons), from micro-scale X-ray CT images of multidirectional CFRP composites, which are then used to generate finite element meshes. The new fibre tracking algorithm combines an ultimate eroding point (UEP) approach with a new Bayesian filtering and tracking approach and offers the following advantages:

- The identification of fibre centre points is enhanced by using a combination of various image filters and the image convolution;

- The delineation of the X-ray CT dataset allows identification of fibres in multidirectional CFRP laminates;

- The global Bayesian filter based on overlapping stacks reduces the number of slices for fibre tracking and thus the computational cost, especially in inclined plies;

- The local fibre tracking algorithm based on Bayesian inference updates the coordinates of fibre centrelines incrementally, which is necessary to track inclined fibres; and

- The FE meshes can be built naturally once the fibre centrelines are identified.

Finally, the synchrotron X-ray CT scanning technique used in this study is very promising in visualising and quantifying microscale geometric features of FRP such as the length, tortuosity, orientation and distribution of fibres. Scanning at faster speed of specimens under in-situ 
dynamic loadings will lead to 3D microscale understanding of damage and fracture evolution inside FRP, which will be invaluable for material design and optimisation in many applications.

\section{ACKNOWLEDGEMENTS}

This study is funded by a U.S. Air Force EOARD grant no. FA8655-12-1-2100 and an EPSRC grant no. EP/J019763/1 at University of Manchester. The X-ray CT experiments are conducted at the Diamond-Manchester Branchline I13-2 at Diamond Light Source session no. MT10456-2. The help and assistance of Mr Razvan Vasiliu, Mr Alex Borsciov and Mr Yu Zhen during the experimentation are greatly appreciated.

\section{NOMENCLATURE}

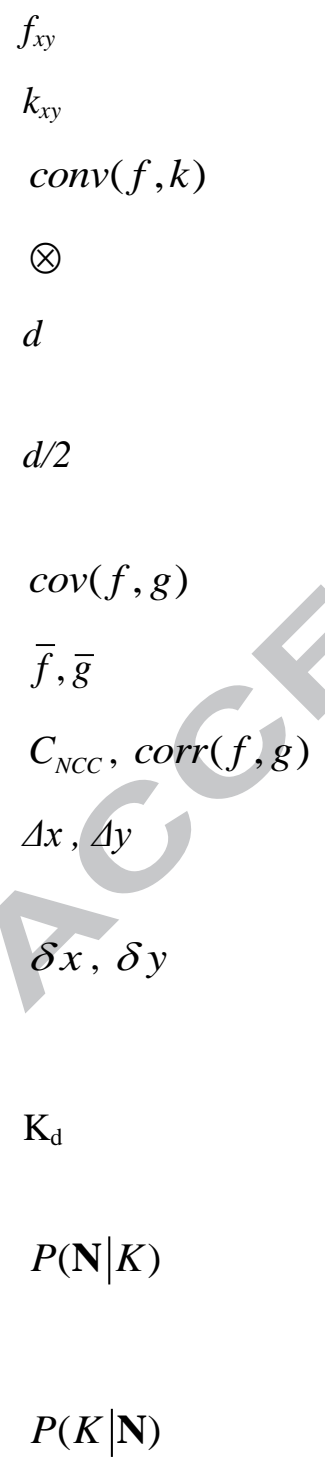

-pixel gray scale intensity at pixel position $(\mathrm{x}, \mathrm{y})$. -processing kernel value corresponding to position $(\mathrm{x}, \mathrm{y})$. -convolution of image $f$ using image processing kernel $k$. -convolution operator that performs a piecewise multiplication. -overlapping stack containing a number of X-ray CT image slices.

-stack overlapping distance measure in number of X-ray CT image slices.

-covariance of two images $f$ and $g$.

-average pixel intensity values of images $f$ and $g$ respectively.

-normalised correlation criterion of two images $f$ and $g$.

-position coordinates at integer pixel precision of matching peak value from correlation of images $f$ and $g$.

-interpolated position coordinates at sub-pixel precision from correlation of images $f$ and $g$ using the neighbouring subsets around a matching peak.

-overlapping stack of slices separated into kernel slices $\mathbf{K}$ and neighbouring-overlapping slices $\mathbf{N}$.

-global prior probability of slices $\mathbf{N}$ to match kernel slices $\mathbf{K}$. This probability is evaluated as a collection of overlapping fibre centres.

-global Bayesian updated probability of slices $\mathbf{K}$ to match 
K

$N$

$P(N \mid K)$

$P(K \mid N)$

neighbouring slices $\mathbf{N}$. This probability is evaluated using a Bayesian inference model.

-kernel slice as a collection of UEPs.

-neighbouring slice as a collection of UEPs.

-local prior probability of UEP coordinates in slice $N$ to match kernel slice $K$. This probability is evaluated as individually on each fibre centre.

-local Bayesian updated probability of UEP coordinates in slice $K$ to match neighbouring slice $N$. This probability is evaluated using a Bayesian inference model.

\section{REFERENCES}

[1] Scott AE, Sinclair I, Spearing SM, Thionnet A, Bunsell AR. Damage accumulation in a carbon/epoxy composite: comparison between a multiscale model and computed tomography experimental results. Composites Part A: Applied Science and Manufacturing. 2012;43(9):151422.

[2] Czabaj MW, Riccio ML, Whitacre WW. Numerical reconstruction of graphite/epoxy composite microstructure based on sub-micron resolution X-ray computed tomography. Composites Science and Technology. 2014;105(0):174-82.

[3] Yu B, Bradley RS, Soutis C, Hogg PJ, Withers PJ. 2D and 3D imaging of fatigue failure mechanisms of 3D woven composites. Composites Part A: Applied Science and Manufacturing. 2015;77:37-49.

[4] Yu B, Blanc R, Soutis C, Withers PJ. Evolution of damage during the fatigue of 3D woven glass-fibre reinforced composites subjected to tension-tension loading observed by time-lapse X-ray tomography. Composites Part A: Applied Science and Manufacturing. 2015.

[5] Nixon-Pearson OJ, Hallett SR. An investigation into the damage development and residual strengths of open-hole specimens in fatigue. Composites Part A: Applied Science and Manufacturing. 2015;69(0):266-78.

[6] Qsymah A, Sharma R, Yang Z and Margetts L. XCT Image-based two-step homogenisation for elastic properties of ultra high performance fibre reinforced concrete considering void sizes, Construction and Building Materials, under review.

[7] Sharma R, Mahajan P, Mittal RK. Fiber bundle push-out test and image-based finite element simulation for 3D carbon/carbon composites. Carbon. 2012;50(8):2717-25.

[8] Ren W, Yang Z, Sharma R, Zhang C, Withers PJ. Two-dimensional X-ray CT image based meso-scale fracture modelling of concrete. Engineering Fracture Mechanics. 2015;133:24-39.

[9] Huang Y, Yang Z, Ren W, Liu G, Zhang C. 3D meso-scale fracture modelling and validation of concrete based on in-situ X-ray Computed Tomography images using damage plasticity model. International Journal of Solids and Structures. 2015;67-68:340-52.

[10] Requena G, Fiedler G, Seiser B, Degischer P, Di Michiel M, Buslaps T. 3D-Quantification of the distribution of continuous fibres in unidirectionally reinforced composites. Composites Part A: Applied Science and Manufacturing. 2009;40(2):152-63. 
[11] Hamad FA-H, Giacomo L, Surya RK. Multi-scale modeling of the elastic response of a structural component made from a composite material using the materials knowledge system. Modelling and Simulation in Materials Science and Engineering. 2012;20(5):055001.

[12] Landi G, Niezgoda SR, Kalidindi SR. Multi-scale modeling of elastic response of threedimensional voxel-based microstructure datasets using novel DFT-based knowledge systems. Acta Materialia. 2010;58(7):2716-25.

[13] Young PG, Beresford-West TBH, Coward SRL, Notarberardino B, Walker B, Abdul-Aziz A. An efficient approach to converting three-dimensional image data into highly accurate computational models2008.

[14] Yang H, Lindquist WB. Three-dimensional image analysis of fibrous materials. 2000. p. 275-82.

[15] Coindreau O, Mulat C, Germain C, Lachaud J, Vignoles GL. Benefits of X-Ray CMT for the Modeling of C/C Composites. Advanced Engineering Materials. 2011;13(3):178-85.

[16] Vignoles GL, Donias M, Mulat C, Germain C, Delesse J-F. Simplified marching cubes: An efficient discretization scheme for simulations of deposition/ablation in complex media. Computational Materials Science. 2011;50(3):893-902.

[17] Coindreau O, Vignoles G, Cloetens P. Direct 3D microscale imaging of carbon-carbon composites with computed holotomography. Nuclear Instruments and Methods in Physics Research Section B: Beam Interactions with Materials and Atoms. 2003;200(0):308-14.

[18] Viguié J, Latil P, Orgéas L, Dumont PJJ, Rolland du Roscoat S, Bloch JF, et al. Finding fibres and their contacts within 3D images of disordered fibrous media. Composites Science and Technology. 2013;89(0):202-10.

[19] Latil P, Orgéas L, Geindreau C, Dumont PJJ, Rolland du Roscoat S. Towards the 3D in situ characterisation of deformation micro-mechanisms within a compressed bundle of fibres. Composites Science and Technology. 2011;71(4):480-8.

[20] Soltani P, Johari MS, Zarrebini M. Effect of 3D fiber orientation on permeability of realistic fibrous porous networks. Powder Technology. 2014;254(0):44-56.

[21] Whitacre WW, Czabaj MW. Automated 3D Digital Reconstruction of Fiber Reinforced Polymer Composites. AIAA Guidance, Navigation, and Control Conference: American Institute of Aeronautics and Astronautics; 2015.

[22] ImageJ. Opensource Software Version 1.60 - Public License at URL: https://imagej.nih.gov/ij/.

[23] Rau C, Wagner U, Pešić Z, De Fanis A. Coherent imaging at the Diamond beamline I13. physica status solidi (a). 2011;208(11):2522-5.

[24] Diamond. http://www.diamond.ac.uk/Home.html. 2016.

[25] Basham M, Filik J, Wharmby MT, Chang PCY, El Kassaby B, Gerring M, et al. Data Analysis WorkbeNch (DAWN). Journal of Synchrotron Radiation. 2015;22(3):853-8.

[26] Wu X, Zhang J, Huang X, Liu D. Separable convolution template (SCT) background prediction accelerated by CUDA for infrared small target detection. Infrared Physics \& Technology. 2013;60:300-5.

[27] Wang J, Kobayashi E, Sakuma I. Coarse-to-fine dot array marker detection with accurate edge localization for stereo visual tracking. Biomedical Signal Processing and Control. 2015;15:49-59. 
[28] Habibi M, Bafandeh A, Montazerolghaem MA. A digital array based bit serial processor for arbitrary window size kernel convolution in vision sensors. Integration, the VLSI Journal. 2014;47(4):417-30.

[29] Epanechnikov V. Non-Parametric Estimation of a Multivariate Probability Density. Theory of Probability \& Its Applications. 1969;14(1):153-8.

[30] Comte F, Genon-Catalot V. Convolution power kernels for density estimation. Journal of Statistical Planning and Inference. 2012;142(7):1698-715.

[31] Ferreira T, Rasband W. The ImageJ User Guide, 2011;1.44.

[32] Sencu RM, Yang Z, Wang YC. From micro to macro: simulating crack propagation in carbon fibre composites. Structural Integrity and Durability of Advanced Composites: Elsevier; 2015.

[33] Sencu RM, Yang Z and Wang YC. A multi-scale stochastic method for crack growth modelling in heterogeneous quasi-brittle materials under uniaxial tension. Engineering Fracture Mechanics. 2016;163:499-522.

[34] Belytschko T (2007). Research directions in computational and composite mechanics. A report of the United States National Committee on theoretical and applied mechanics. 